\title{
Anti-Inflammatory Effects of p-Coumaric Acid, a Natural Compound of Oldenlandia diffusa, on Arthritis Model Rats
}

\author{
Hao Zhu ${ }^{(D)},{ }^{1}$ Qing-hua Liang, ${ }^{2}$ Xin-gui Xiong $\left(\mathbb{D},{ }^{2}\right.$ Yang Wang, ${ }^{2}$ Zhi-hui Zhang, \\ Mei-juan Sun, ${ }^{1}$ Xun $L{ }^{3}{ }^{3}$ and Dan $W^{4}{ }^{4}$ \\ ${ }^{1}$ Institute of Combined Traditional Chinese and Western Medicine, The First Affiliated Hospital of Soochow University, Suzhou, \\ Jiangsu 215006, China \\ ${ }^{2}$ Institute of Combined Traditional Chinese and Western Medicine, Xiangya Hospital, Central South University, Changsha, \\ Hunan 410008, China \\ ${ }^{3}$ Institute of Combined Traditional Chinese and Western Medicine, Suzhou Municipal Hospital, Soochow, Jiangsu 215008, China \\ ${ }^{4}$ Institute of Combined Traditional Chinese and Western Medicine, The 4th Hospital of Changsha, Changsha, Hunan 410008, China
}

Correspondence should be addressed to Xin-gui Xiong; xiongxg07@gmail.com

Received 23 August 2017; Accepted 1 January 2018; Published 22 February 2018

Academic Editor: Jairo Kennup Bastos

Copyright (C) 2018 Hao Zhu et al. This is an open access article distributed under the Creative Commons Attribution License, which permits unrestricted use, distribution, and reproduction in any medium, provided the original work is properly cited.

Objectives. In China, Oldenlandia diffusa (OD) is a natural herb that is widely used and has been proven to be effective in the treatment of rheumatoid arthritis (RA). This study aimed to preliminarily reveal the mechanism by which OD exerts its beneficial effect. Methods. Ultra-performance liquid chromatography photodiode array was applied to identify the absorbable compounds in the plasma of collagen-induced arthritis (CIA) model rats. After 2 weeks, an OD decoction or the identified absorbable compound was administered to CIA rats. Morphology, X-ray images of the joints, pathological images, arthritis index, and cytokine (TNF$\alpha$ and IL-6) levels were evaluated. Results. p-Coumaric acid (p-CA) was identified as the absorbed compound in plasma. After administration of p-CA solution or the OD decoction, symptoms in the treated rats were alleviated as compared to the untreated model rats, and inflammatory cell infiltration was suppressed. The arthritis index and serum levels of TNF- $\alpha$ and IL-6 were decreased as compared to the control group. Conclusions. OD may exert its anti-inflammatory effect on RA via its active ingredient, p-CA. This information sheds light on the mechanism by which OD exerts its anti-inflammatory effort in RA and forms the basis for further development of therapeutic agents for RA.

\section{Introduction}

Rheumatoid arthritis (RA) is a heterogeneous systemic autoimmune disease that impairs the quality of life of patients [1] and presents with different symptoms [2]. Exterior changes involve joint malformation, swelling, and joint dysfunction $[3,4]$, while interior pathological changes mainly involve synovial hyperplasia and cartilage destruction. All of these changes are accompanied by systemic inflammatory responses $[5,6]$, involving many cytokines, such as TNF- $\alpha$ $[7,8]$ and IL-6 [9-11], which are responsible for initiating, propagating, and maintaining inflammation [12] in a complex network [13]. Recent studies have shown that TNF- $\alpha$ governs the crosstalk between fibroblast-like synoviocytes and chondrocytes [12] and might regulate the expressional changes in matrix-degrading enzymes and inflammatory mediators in fibroblast-like synoviocytes [12] and induce the inflammatory responses. TNF- $\alpha$ also governs the crosstalk between fibroblast-like synoviocytes and chondrocytes [12] and consequently induce inflammatory responses. IL- 6 also plays a key role in RA. IL-6 can induce expression of vascular endothelial growth factor (VEGF) in synovial fibroblasts and other cells [14], promoting pannus formation in the synovium and causing an inflammatory response [14]. IL-6 is also implicated in damage to the articular cartilage in RA [15] and promotes further recruitment of leukocytes and inflammatory responses in the joints of RA patients [15]. Taken together, it is clear that inflammation plays an important role in the pathology of RA. 
In China, traditional herbs are used as medication to treat patients with RA [16]. In traditional Chinese medicine, principles of yin-yang, the five elements, zang, and viscera guidelines are used [17]. Accordingly, prescriptions consisting of multiple herbs are used to treat RA patients, with many patients experiencing marked beneficial effects [18]. However, neither the mechanism of the complex traditional Chinese prescription, nor the active ingredients in these medications remain unknown; yet, such knowledge would be immensely valuable. Considering that one prescription is usually formulated from multiple herbs, research on such complex decoctions is complicated, and therefore it is convenient and advantageous to focus on a single herb when attempting to elucidate the mechanisms and active compounds.

Among the herbs traditionally used, Oldenlandia diffusa (OD) is a key component of herbal prescriptions for RA. Shan et al. [19] proved that OD has immunomodulating activity in vitro. Moreover, it was shown that OD could inhibit the production of TNF- $\alpha$, IL-6, PGE-2, COX-2, and iNOS [20] and might exert anti-inflammatory effects via downregulation of TNF- $\alpha$ and IL-6 [20, 21]. These 2 cytokines are proinflammatory factors that could activate NF$\kappa \mathrm{B}$ signaling $[22,23]$, thereby increasing the inflammatory response [24]. We have also previously shown that $\mathrm{OD}$ has an anti-inflammatory effect on collagen-induced arthritis (CIA) [25]. Considering that OD contains multiple ingredients, such as ursolic acid, oleanolic acid, kaempferol, p-CA, ferulic acid, rutin, scopolamine lactone, and caffeic acid [26], which of these ingredients are responsible for its effects is unknown. In this study, therefore, we investigated the active ingredients of OD further.

\section{Methods}

2.1. Ethics Statement. All protocols involving animals in this study were approved by the Ethics Committee of Central South University (Grant number 20120435), China. Our experiment was performed in accordance with the Guidance for the Humane Care and Treatment of Laboratory Animals published by the Ministry of Science and Technology of China.

2.2. Identification of Absorbable Compounds in OD. We used ultra-performance liquid chromatography (UPLC) to identify the compounds in OD that can be absorbed in rat plasma. We employed a chromatography column (ACQUITY UPLC C18; $2.1 \times 50 \mathrm{~mm} ; 1.7 \mu \mathrm{m})$, at a column temperature of $38^{\circ} \mathrm{C}$, wavelength of $180-360 \mathrm{~nm}$, flow rate of $0.8 \mathrm{~mL} / \mathrm{min}$, and a sample volume of $8 \mu \mathrm{l}$. Analysis time was $15 \mathrm{~min}$. The reference standards (i.e., oleanolic acid, kaempferol, ursolic acid, p-CA, ferulic acid, scopolamine lactone, rutin, and caffeic acid) were purchased from Shanghai Yuanye BioTechnology Co., Ltd., Shanghai, China. Acetonitrile and methanol (HPLC grade) were purchased from the Tedia Company, Inc. (Fairfield, OH, USA). The herbal drug OD was purchased from TCM Dispensary, Xiangya Hospital, Changsha, China. We ground OD into powder and mixed it with purified water in a ratio of $1 \mathrm{~g}$ of powder to $8 \mathrm{ml}$ of water. After boiling for $30 \mathrm{~min}$, we filtered the decoction, condensed the liquid in a vacuum, at $60^{\circ} \mathrm{C}$, and dehydrated it into powder. Next, we dissolved the freeze-dried OD powder in purified water in a ratio of $1 \mathrm{~g}$ of powder to $10 \mathrm{ml}$ of water, followed by centrifugation ( $1200 \mathrm{r} / \mathrm{min}, 15 \mathrm{~min})$, after which we extracted and filtered $(0.45 \mu \mathrm{m}$ filter $)$ the supernatant. The yield rate was $43.1 \mathrm{mg}$ (crude drug)/ml. We prepared the previous 8 reference standard compounds in solution by dissolving in methanol at concentrations of $0.167 \mathrm{mg} / \mathrm{ml}$, $0.2 \mathrm{mg} / \mathrm{ml}, 0.163 \mathrm{mg} / \mathrm{ml}, 0.187 \mathrm{mg} / \mathrm{ml}, 0.16 \mathrm{mg} / \mathrm{ml}, 0.11 \mathrm{mg} / \mathrm{ml}$, $0.168 \mathrm{mg} / \mathrm{ml}$, and $0.232 \mathrm{mg} / \mathrm{ml}$, respectively. We then mixed all these in a ratio of $1: 1: 1: 1: 1: 1: 1: 1$ to create a test solution. All solutions were sealed and then stored at a temperature of $4^{\circ} \mathrm{C}$.

Sprague-Dawley (SD) rats weighing $150 \pm 30 \mathrm{~g}$ were obtained from the Laboratory of Hunan Provincial People's Hospital. Rats were divided into 2 groups, that is, the OD group $(n=5)$ and the control group $(n=5)$. First, we calculated the standard human daily herbal dose and converted it to a rat daily herbal dose according to the body surface area of an individual weighing $70 \mathrm{~kg}$ [16]; the daily dose per rat was thus $2.7 \mathrm{mg} / \mathrm{g}$ (crude drug/weight). We administered the OD decoction orally at the calculated dose to the OD group and administered the same volume of purified water to the control group. Three days later, we decapitated all rats and extracted their plasma, mixed the plasma with acetonitrile, ethyl acetate, and acetone at a ratio of $1: 2: 1: 0.6$ ( $\mathrm{vol}: \mathrm{vol}: \mathrm{vol}$ ), and centrifuged the mixture (1200 r/min, $15 \mathrm{~min})$. We extracted and filtered the supernatant, air dried it under nitrogen gas, redissolved and recentrifuged the sample as before, and stored it as a test sample.

2.3. Administration of $O D$ and $p-C A$. Rats were randomly selected and divided into 2 groups: a control $(n=20)$ and a replication $(n=60)$ group, in which collagen-induced arthritis was generated. First, we prepared bovine collagen type II (BIIC) solutions with complete Freund's adjuvant and incomplete Freund's adjuvant, respectively. These 2 different solutions were subcutaneously injected into the tail, back, and soles of each rat on the 1st and 7th day, respectively. After 14 days, we randomly divided the replication group into an OD group, a p-CA group, and a model group. Based on the results of the previous chromatographic analysis, we calculated the p-CA content in OD and converted this to a rat dose [16]. Based on the normal human daily herbal dose, we calculated the daily dose of p-CA for a rat, which was $13.8 \mu \mathrm{g} / \mathrm{g}$. Then, we used oral gavage to administer the OD decoction $(1.35 \mathrm{mg} / \mathrm{g}$ twice a day) to the OD group and p-CA solution $(6.91 \mu \mathrm{g} / \mathrm{g}$, twice a day) for the p-CA group. The model group was given purified water (the same volume as in the other groups; twice a day). Rats in the normal group received no intervention.

2.4. Morphological Analysis. On the 1st, 14th, 21st, 28th, 35 th, and 42nd day, we observed and measured the arthritis index (AI) $[27,28]$ of each rat's posterior limb. According to the degree of joint redness and swelling, as well as joint enlargement and deformity, we scored every symptom on a scale of 0 to 4 points, with 0 meaning no arthritis, 1 meaning 


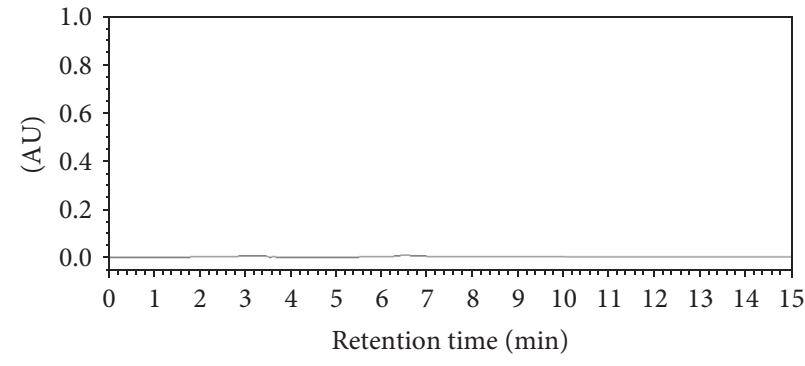

(a)

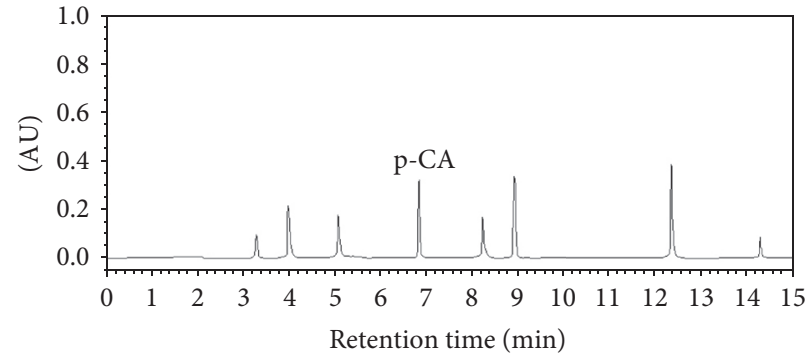

(b)

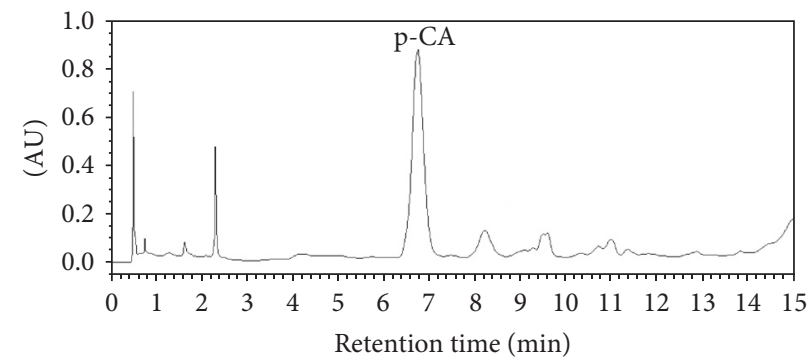

(c)

FIGURE 1: Chromatogram of each group (308 nm): (a) blank methanol; (b) 8 reference compounds; (c) test solution of OD. p-CA refers to p-coumaric acid.

mild swelling plus appearance of red spots, 2 meaning moderate swelling of joints, 3 meaning severe swelling of joints, and 4 meaning severe swelling of joints and inability to bear weight.

2.5. Radiographic Changes in Rats' Joints. On days 0, 14, 28, and $42, \mathrm{X}$-ray images of the right rear foot of each rat were obtained to observe joint changes. We used a diagnostic Xray machine (PHILIPS, Inc., Andover, MA, USA) to take the radiographs.

2.6. Pathological Changes in Rats' Joints. After injection of Freund's adjuvant with BIIC, the posterior limbs of the rats were amputated on days 14,28 , and 42 . The p-CA group and $\mathrm{OD}$ group were divided after the 28th day, and thus the joints of these rats were amputated on days 28 and 42. All these limbs were stored in formalin solution, at $4^{\circ}$. Finally, we removed the synovium from each joint; all of the synovia was stored and sealed in formalin. After preparation, these samples were embedded in dehydrated paraffin and sliced into sections in $4 \mu \mathrm{m}$ and these were stained with hematoxylin-eosin (HE) as previously described [25]. Pathological changes were observed by microscope (magnified 10x; Leica DFC425C; Leica, Wetzlar, Germany).

2.7. TNF- $\alpha$ and IL-6 Levels. On the 28th and 42nd day, rats were decapitated and their serum was obtained. TNF- $\alpha$ and IL-6 levels were tested and compared using enzyme-linked immunosorbent assays (ELISA Cusabio Biotech Co., Ltd., Wuhan, China), as previously described [25].

2.8. Statistical Analyses. The data of the arthritis index and TNF- $\alpha$ and IL- 6 levels were statistically analyzed using SPSS 15.0 and compared among groups.

\section{Results}

3.1. Identification of the Active Compounds in $O D$. In the preliminary experiment, we detected each reference separately, to find the chromatographic wavelength and showtime of each reference standard. Compared with the wavelength and showtime of each reference, as shown in Figure 1, the 8 reference standards were well separated and identified as oleanolic acid $(224 \mathrm{~nm}, 3.29 \mathrm{~min})$, kaempferol $(366 \mathrm{~nm}$, $4.02 \mathrm{~min})$, ursolic acid $(223 \mathrm{~nm}, 5.09 \mathrm{~min}), \mathrm{p}-\mathrm{CA}(308 \mathrm{~nm}$, $6.81 \mathrm{~min})$, ferulic acid $(321 \mathrm{~nm}, 8.21 \mathrm{~min})$, scopolamine lactone $(228 \mathrm{~nm}, 8.92 \mathrm{~min})$, rutin $(255 \mathrm{~nm}, 12.36 \mathrm{~min})$, and caffeic acid $(325 \mathrm{~nm}, 14.23 \mathrm{~min})$. When analyzing the OD solution, we observed a large peak at a wavelength of $308 \mathrm{~nm}$ at $6.79 \mathrm{~min}$ (Figure 1(c)) in the OD solution. In the blank methanol (Figure 1(a)), there was no peak around $6.79 \mathrm{~min}$. We speculate that the large peak in OD group's chromatogram might be p-CA (Figure 1(c)).

3.2. Identification of Absorbable Compounds in OD. Compared with the wavelength and showtime of each reference, as showed in Figure 2(b), the 8 reference standards were well separated and identified as oleanolic acid (224 nm, $3.31 \mathrm{~min})$, kaempferol $(366 \mathrm{~nm}, 3.98 \mathrm{~min})$, ursolic acid $(223 \mathrm{~nm}$, $5.13 \mathrm{~min}), \mathrm{p}-\mathrm{CA}(308 \mathrm{~nm}, 6.81 \mathrm{~min})$, ferulic acid $(321 \mathrm{~nm}$, $8.18 \mathrm{~min})$, scopolamine lactone $(228 \mathrm{~nm}, 8.90 \mathrm{~min})$, rutin $(255 \mathrm{~nm}, 12.34 \mathrm{~min})$, and caffeic acid (325 nm, $14.21 \mathrm{~min})$. After orally administering the OD decoction to rats, the plasma was tested to identify which compounds were absorbed by the rats. As shown in Figure 2(c), a large peak was well separated at a wavelength of $308 \mathrm{~nm}$ at $6.80 \mathrm{~min}$ from the OD rat plasma, while blank plasma (Figure 2(a)) showed no such peak around $6.81 \mathrm{~min}$. We speculate that the large peak in the plasma's chromatogram might be $\mathrm{p}-\mathrm{CA}$ (Figure 2(c)). 


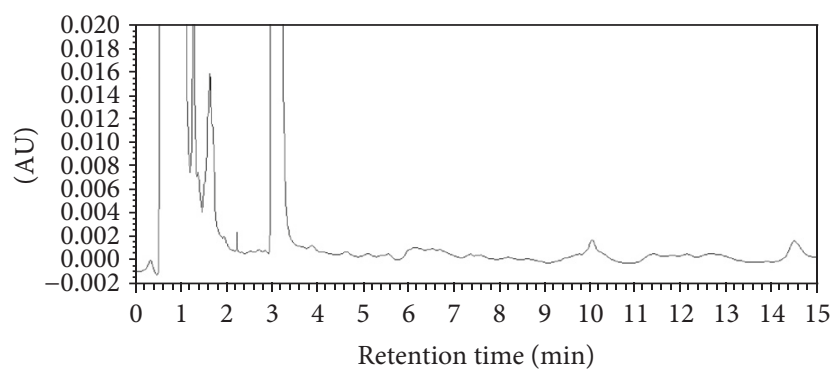

(a)

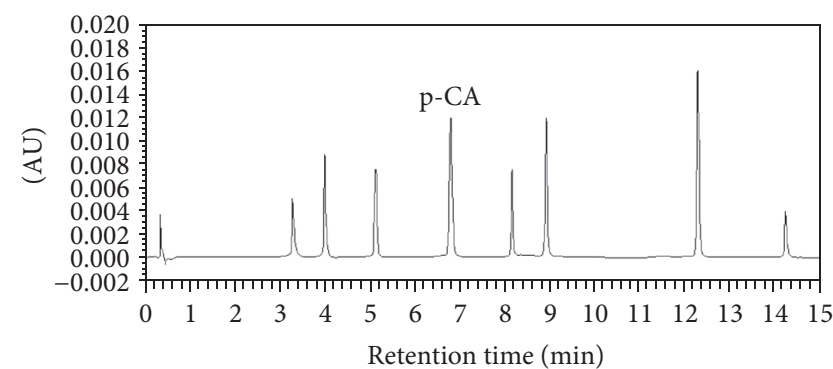

(b)

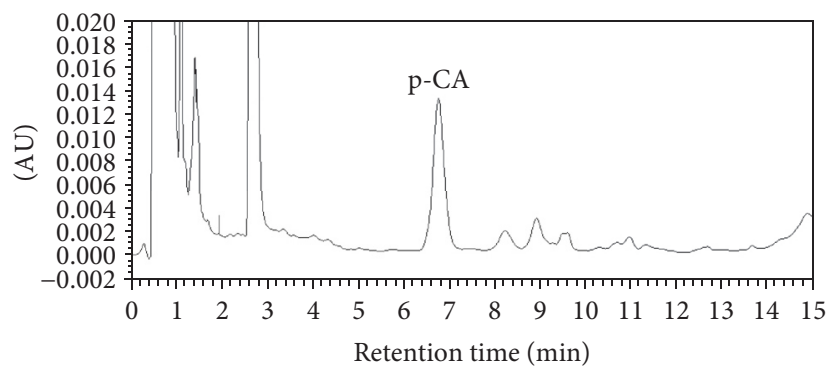

(c)

FIGURE 2: Chromatogram of each group ( $308 \mathrm{~nm}$ ): (a) plasma without added compounds; (b) 8 reference compounds; (c) the plasma of rats that had been given OD decoction by oral gavage. $\mathrm{p}$-CA refers to $\mathrm{p}$-coumaric acid.

3.3. Morphological Analysis. Before BIIC injection, as shown in Figure 3(a), in the control group, the posterior limb remained slim and flexible. After the injection, morphological changes occurred within 4-6 days. In the model group, from the 14th day to the 42 nd day (Figures $3(\mathrm{~b})-3(\mathrm{~d})$ ), the ankle and toe joints of the rats gradually showed more swelling, redness, ankylosis, and hyperemia. This was particularly notable by the 42 nd day (Figure $3(d)$ ). Simultaneously, rats in the model group became inactive and drowsy and ate and drank less. After treatment with p-CA (Figures 3(e) and $3(\mathrm{f})$ ), at the 28th day in the p-CA group (Figure 3(e)), there were minor changes in terms of skin shrinking and alleviation of swelling, as compared to the model group (Figure 3(c)). By the 42nd day (Figure 3(f)), ankle swelling and skin redness were notably alleviated as compared to the model group (Figure 3(d)). Similarly, after administration of the OD decoction (Figures 3(g) and 3(h)), as shown in the OD group by the 28th day (Figure $3(\mathrm{~g})$ ), the ankle showed a mild change in terms of skin shrinking and alleviation of swelling, as compared to the model group. By the 42nd day (Figure 3(h)), ankle swelling and skin redness were markedly alleviated as compared to the model group.

3.4. Radiographic Analysis of Rats' Joints. X-ray images showed pathological changes inside the joints. As shown in Figure 4(a), the control group showed no swelling; both toe joints and ankle joints showed clear space. After BIIC injection, radiographic changes occurred within 4-6 days. In the model group, from the 14th day to the 42nd day (Figures 4(b)-4(d)), the toe joint space and ankle joint space gradually disappeared or became indistinct, and swelling of the surrounding tissue gradually worsened. By the 42 nd day (Figure $4(\mathrm{~d})$ ), some toe joints even showed joint fusion and loss of function. After administration of p-CA, from the 28th day to the 42 nd day (Figures $4(\mathrm{e})$ and $4(\mathrm{f})$ ), the space in the ankle joint of the p-CA group gradually became clear as compared to the model group (Figures 4(c) and 4(d)). After treatment with OD decoction, from the 28th day to the $42 \mathrm{nd}$ day (Figures $4(\mathrm{~g})$ and $4(\mathrm{~h})$ ), the same radiographic changes as seen in the $\mathrm{p}$-CA group were even more obvious in the OD group, as compared to the model group (Figures 4(c) and $4(\mathrm{~d})$ ). The tissue swelling in the p-CA and OD group by the 42nd day (Figures 4(f) and 4(h)) showed marked alleviation, as compared with the model group (Figure 4(d)). All posterior limbs were functional by the 42 nd day after administration of p-CA or OD decoction (Figures $4(\mathrm{f})$ and $4(\mathrm{~h}))$. The $\mathrm{p}-\mathrm{CA}$ and OD groups thus showed a considerable amelioration of arthritic ankle joints.

3.5. Pathologic Changes in Rats' Joints. As shown in Figure 5(a), mild inflammatory cell infiltration was observed in the control group. As compared with the control group, the inflammatory cell infiltration deteriorated from the 14th day (Figure 5(b)) to the 42nd day (Figure 5(d)) in the model group. The infiltration became more concentrated during the course in the model group and was particularly notable on the 28th day (Figure 5(c)) and 42nd day (Figure 5(d)). We then treated the p-CA group on the 14th day; 2 weeks later, that is, on the 28th day, as shown in Figure 5(e), there was no major change in inflammatory cell infiltration, as compared with the model group at the time point (Figure 5(d)). After another 2 weeks, that is, at the 42 nd day, in the p-CA group (Figure 5(f)), inflammatory cell infiltration was alleviated as compared with the model group (Figure $5(\mathrm{~d})$ ), as well as with the inflammatory cell infiltration 2 weeks earlier (Figure 5(e)). Two weeks after treatment of the OD group 


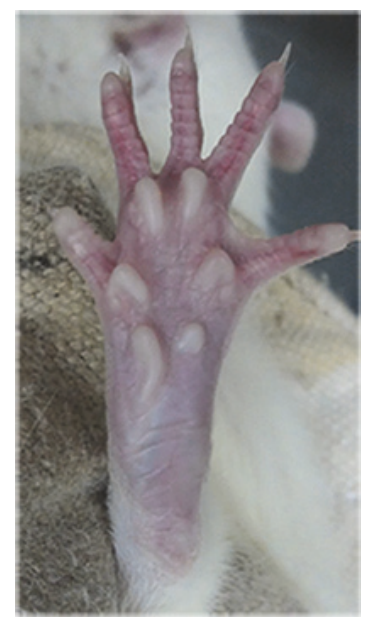

(a)

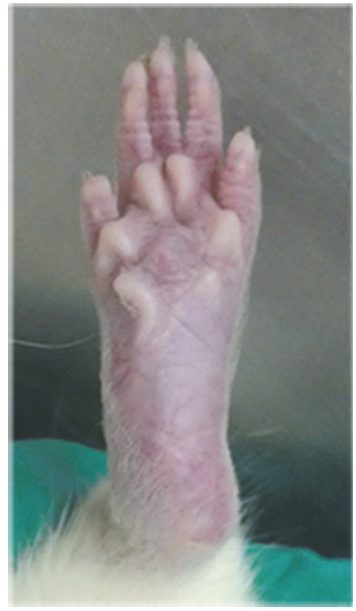

(e)

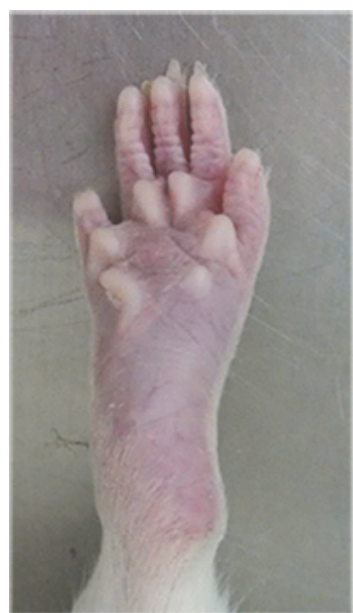

(b)

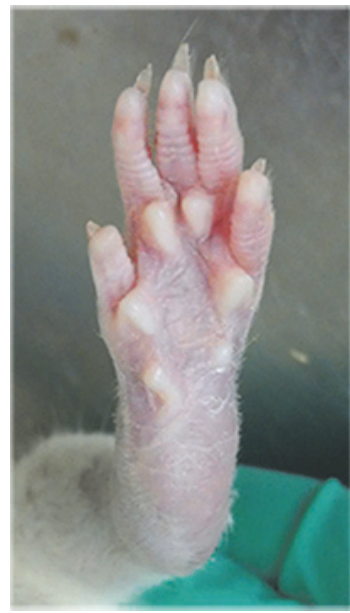

(f)

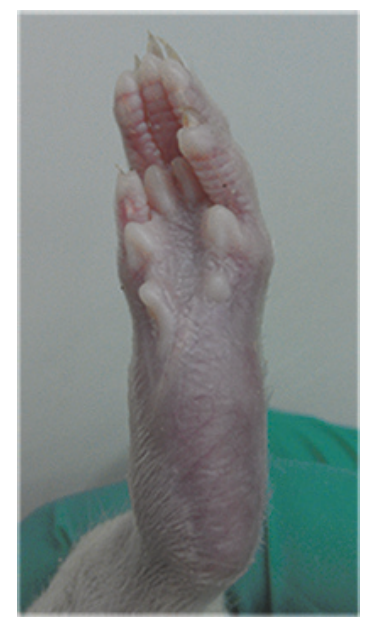

(c)

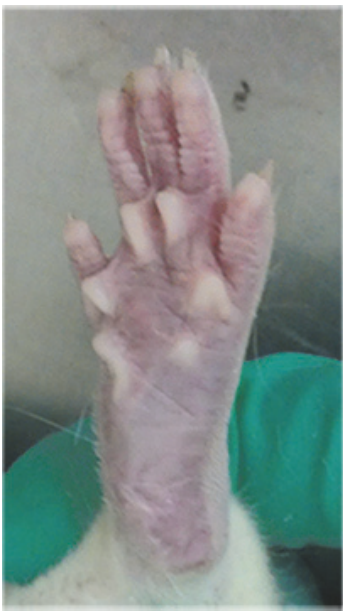

(g)

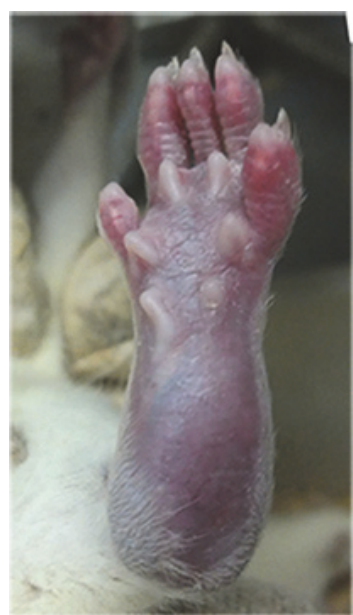

(d)

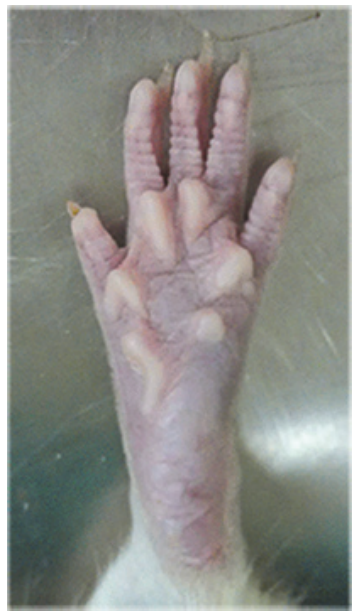

(h)

Figure 3: Morphological changes of joints in each group, from the 1st day to the 42nd day. (a) Posterior limb of a rat in the normal group. (b) Posterior limb of a rat in the model group, on the 14th day. (c) Posterior limb of a rat in the model group, on the 28th day. (d) Posterior limb of a rat in the model group, on the 42 nd day. (e) Posterior limb of a rat treated with p-CA, on the 28th day. (f) Posterior limb of a rat treated with p-CA, on the 42nd day. (g) Posterior limb of a rat treated with OD decoction, on the 28th day. (h) Posterior limb of a rat treated with OD decoction, on the 42nd day.

on the 14th day, that is, on the 28th day, as shown in Figure $5(\mathrm{~g})$, there was no major change in inflammatory cell infiltration as compared with the model group at the time (Figure 5(c)). After another 2 weeks, that is, on the 42nd day, the inflammatory cell infiltration in the OD group (Figure 5(h)) was alleviated as compared with the model group (Figure 5(d)), as well as with the infiltration 2 weeks earlier (Figure 5(g)).

3.6. Analysis of Arthritis Index. Before intervention, as shown in Figure 5, each group was scored as 0 on the AI. The control group had a score of 0 throughout the experiment. After injection, from the 14th day to the 21st day, the index of the other 3 groups started to increase and was not significantly different among the model, p-CA, and OD groups. From the 28th day to the 42nd day, the index of the model group stabilized at a high value, whereas both the p-CA group and the OD group had lower index values than the model group $(p<0.05)$, which continuously decreased from the 21st day to the 42 nd day. On the 28th day, the index of the OD group appeared to be lower than that of the p-CA group, but without statistical significance. On the 35th and particularly on the 42 nd day, the OD group had a lower AI than the p-CA group $(p<0.05)$.

3.7. Analysis of TNF- $\alpha$ and IL-6 Levels. According to the ELISA results shown in Figure 6, TNF- $\alpha$ levels in the model group rose from the 28th day to the 42 nd day and were markedly higher than those in the control group. After intervention, on both the 28th day and the 42nd day, the p-CA group had lower levels than the model group $(p<$ 0.05 ), although these values were still higher than those in the control group. The level of TNF- $\alpha$ in the OD group decreased from the 28 th day to the 42 nd day and was lower than those in the model group $(p<0.05)$. Additionally, as shown in Figure 6, the IL-6 levels in the model group increased from 


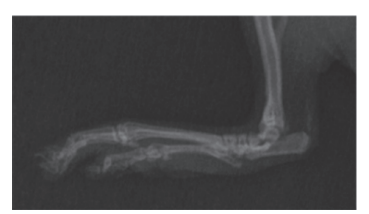

(a)

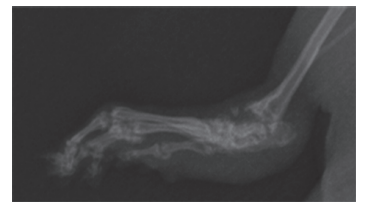

(e)

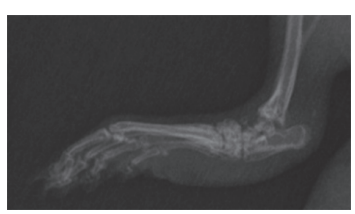

(b)

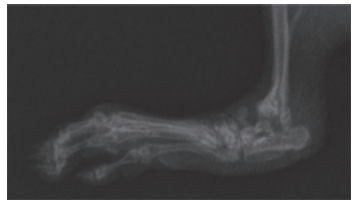

(f)

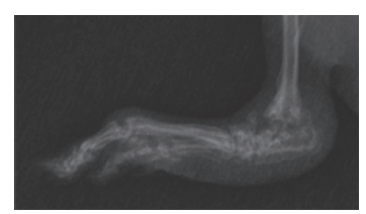

(c)

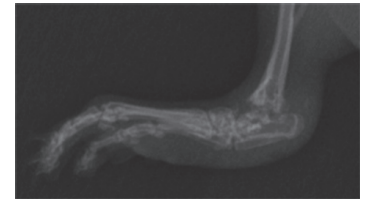

(g)

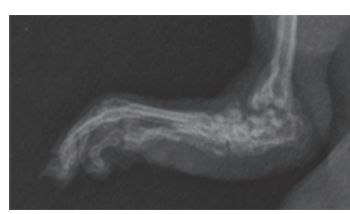

(d)

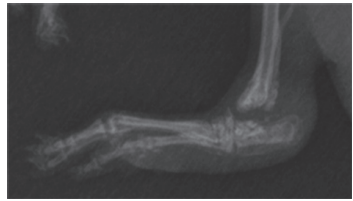

(h)

Figure 4: X-ray film of the joints in each group, from the 1st day to the 42nd day. (a) Posterior limb of a rat in the normal group. (b) Posterior limb of a rat in the model group, on the 14th day. (c) Posterior limb of a rat in the model group, on the 28th day. (d) Posterior limb of a rat in the model group, on the 42nd day. (e) Posterior limb of a rat treated with p-CA, on the 28th day. (f) Posterior limb of a rat treated with p-CA, on the 42 nd day. (g) Posterior limb of a rat treated with OD decoction, on the 28 th day. (h) Posterior limb of a rat treated with OD decoction, on the 42 nd day.

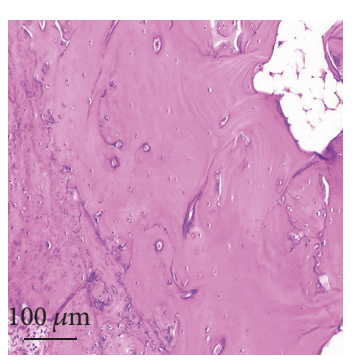

(a)

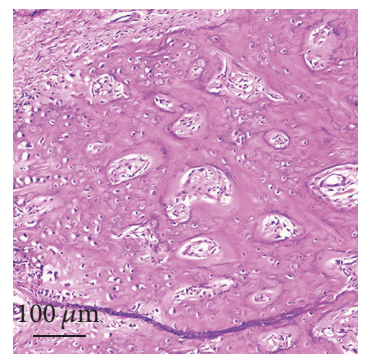

(e)

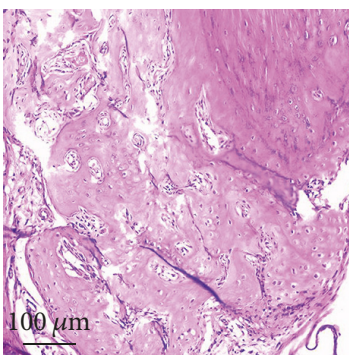

(b)

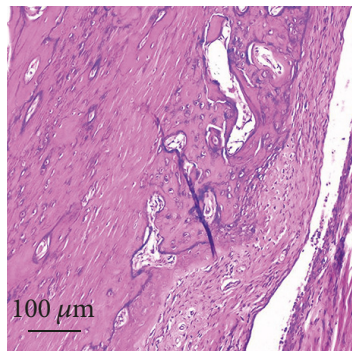

(f)

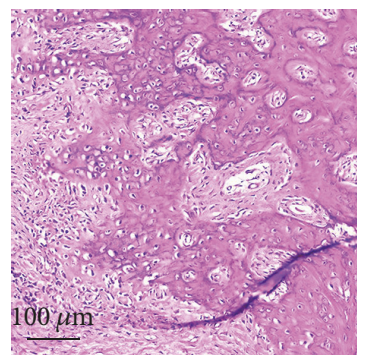

(c)

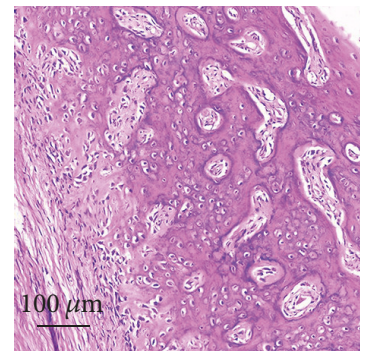

(g)

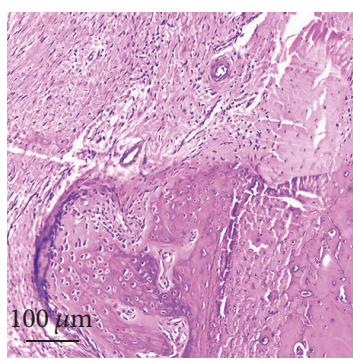

(d)

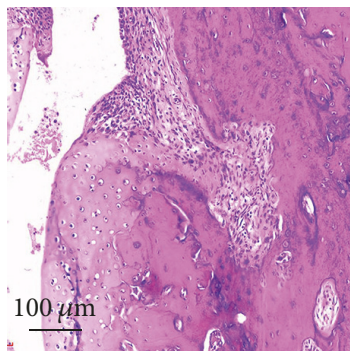

(h)

FIGURE 5: Synovium pathomorphology as seen by hematoxylin-eosin-staining in each group, from the 1st day to the 42nd day. (a) Normal group. (b) Model group, 14th day. (c) Model group, 28th day. (d) Model group, 42nd day. (e) p-CA group, 28th day. (f) p-CA, 42nd day. (g) OD group, 28th day. (h) OD group, 42nd day.

the 28th day to the 42nd day and were markedly higher than those in the control group. After intervention, on both the 28 th day and the $42 \mathrm{nd}$ day, the levels in the p-CA group were lower than those in the model group $(p<0.05)$ but remained higher than those in the control group. The level of IL- 6 in the OD group decreased from the 28th day to the 42 nd day and was lower than those in the model group $(p<0.05)$.

\section{Discussion}

In China, OD has been widely used for treating RA, and multiple components in $\mathrm{OD}$, including $\mathrm{p}$-CA, have been identified [29, 30]. p-CA is one of the active ingredients in OD and could prevent cell-mediated immune responses in rats [31] and decrease the expression of the inflammatory mediators TNF- $\alpha$ and IL-6 [31] and circulating immune complexes in adjuvant-induced arthritic rats [31]. Using UPLC, we verified that p-CA is one of the ingredients in OD (Figure 1) and that it could be absorbed into rat's blood after intragastric administration of OD decoction (Figure 2). Based on these findings, we administered p-CA and OD, separately, to CIA model rats. As shown in Figures 3 and 4, CIA led to a range of inflammatory features, including joint swelling, hyperemia, joint fusion, and loss of function. After 


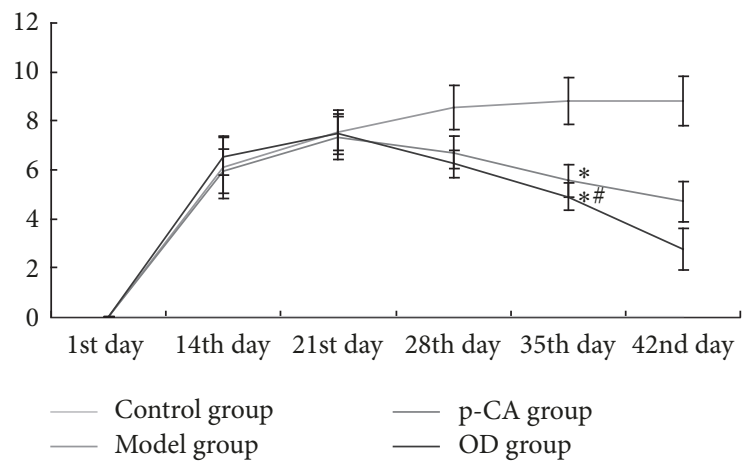

FIGURE 6: Arthritis index (AI) in each group (means $\pm \mathrm{SD}$ ), from the 1 st day to the $42 \mathrm{nd}$ day. From the 28 th day to the $42 \mathrm{nd}$ day, the pCA group and OD group showed statistically significant differences $\left({ }^{*} p<0.05\right)$ as compared to the model group. On the 42nd day, the OD group showed a significant difference $\left({ }^{\#} p<0.05\right)$ as compared with the p-CA group.

treatment with p-CA or OD, these symptoms were alleviated, including skin shrinking, alleviation of swelling, clearing of the joint space, and improvement of the range of motion. Thus, p-CA and OD could alleviate CIA symptoms. The change in AI (Figure 6) also demonstrated that both p-CA and OD could downregulate CIA symptoms. These findings strongly indicate that OD might alleviate CIA symptoms via its ingredient, p-CA.

Subsequent experiments shed light on how p-CA or OD exerted these effects. TNF- $\alpha$ and IL- 6 are well-known cytokines that play important roles in the pathogenesis of RA [32]. TNF- $\alpha$ is released from macrophagocytes in the synovium and pannus and may have potent antioxidant and anti-inflammatory effects [33]. IL-6 can trigger the immune system by activating B-cells [34], releasing immunoglobulins, and increasing the production of rheumatoid factor [35, 36]. It could also enhance the production of TNF- $\alpha$ and IL$1 \beta[15]$, thereby aggravating cartilage destruction $[15,37]$. Recent studies have shown that TNF- $\alpha$ and IL- 6 could induce oxidative stress by initiating the NF- $\kappa \mathrm{B}$ activation cascade [38], causing erosion of the articular cartilage as well as bone destruction $[39,40]$. In this study, we showed that the serum levels of TNF- $\alpha$ and IL-6 increased markedly in the CIA rat model of RA, and after treatment with p-CA or OD these cytokine levels decreased (Figure 7). This may indicate that both p-CA and OD could suppress TNF- $\alpha$ and IL- 6 and thereby could counter the inflammatory response, such as inflammatory cell infiltration [41]. As shown in Figure 4, the model group had more marked inflammatory cell infiltration than the control group, which was related to the high serum levels of TNF- $\alpha$ and IL- 6 , and after intervention with p-CA or OD the cell infiltration in both groups was alleviated. Thus, both p-CA and OD could suppress the infiltration of inflammatory cells, supporting its anti-inflammatory effect. A previous report also indicated that $\mathrm{p}$-CA could have a moderate inhibitory effect on the activation of NF- $\kappa$ B [42], which could accelerate vascular proliferation, synovial hyperplasia [43], and cytokine proliferation [44, 45]. Pragasam et al. [31] also showed that $\mathrm{p}$-CA may inhibit the expression of the

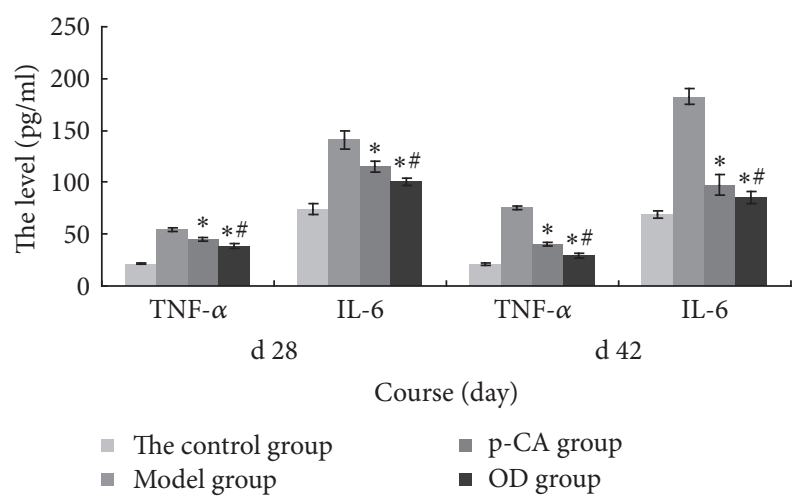

Figure 7: Serum levels of TNF- $\alpha$ and IL-6 in each group (means \pm $\mathrm{SD})$ on the 28th day and 42nd day. The p-CA group and OD group showed statistically significant differences $\left({ }^{*} p<0.05\right)$ as compared with the model group. The OD group also showed a significant difference $\left.{ }^{\#} p<0.05\right)$ as compared with the $\mathrm{p}$-CA group.

NF- $\kappa$ B gene, resulting in reduced TNF- $\alpha$ expression. These results are concordant with our own.

\section{Conclusion}

Our study thus indicated that both p-CA and OD could alleviate the symptoms of arthritis in rats. Considering that $\mathrm{p}-\mathrm{CA}$ is one of the active ingredients in $\mathrm{OD}$, we speculated that OD may exert its anti-inflammatory effects via $\mathrm{p}-\mathrm{CA}$, in a mechanism that included suppression of inflammatory cell infiltration, as well as the levels of TNF- $\alpha$ and IL-6. Given that OD contains multiple ingredients, we speculate that there are additional effective ingredients that play a role in the therapeutic effect of OD, which may have additional targets. Elucidating the mechanisms underlying the effects of OD in RA will therefore require further study. This study has shed light on the mechanism by which OD exerts its antiinflammatory effort in RA and forms the basis for further development of therapeutic agents for RA.

\section{Abbreviations}

$\begin{array}{ll}\text { AI: } & \text { Arthritis index } \\ \text { BIIC: } & \text { Bovine collagen type II } \\ \text { CIA: } & \text { Collagen-induced arthritis } \\ \text { OD: } & \text { Oldenlandia diffusa } \\ \text { p-CA: } & \text { p-Coumaric acid } \\ \text { RA: } & \text { Rheumatoid arthritis } \\ \text { UPLC-PDA: } & \text { Ultra-performance liquid chromatography } \\ & \text { photodiode array. }\end{array}$

\section{Conflicts of Interest}

None of the authors of the paper have any conflicts of interest regarding the publication.

\section{Authors' Contributions}

Qing-hua Liang and Xin-gui Xiong designed and guided the experiment; Hao Zhu wrote the first draft of the paper; Dan 
Wu and Hao Zhu performed the UPLC and rat experiments; Zhi-hui Zhang and Mei-juan Sun documented and analyzed the data. All authors read and approved the final version of the paper.

\section{Acknowledgments}

This project was supported by the National Natural Science Foundation of China (Grant no. 81102564) and a Specialized Research Fund for the Doctoral Program of Higher Education (Grant no. 20110162120004). The authors would like to acknowledge Editage (https://www.editage.com) for English language editing.

\section{References}

[1] G. Gong and J. Mao, "Health-related quality of life among Chinese patients with rheumatoid arthritis: The predictive roles of fatigue, functional disability, self-efficacy, and social support," Nursing Research, vol. 65, no. 1, pp. 55-67, 2016.

[2] P. M. Johnsson and K. Eberhardt, "Hand deformities are important signs of disease severity in patients with early rheumatoid arthritis.," Rheumatology, vol. 48, no. 11, pp. 1398-1401, 2009.

[3] J. F. Fries, P. Spitz, R. G. Kraines, and H. R. Holman, "Measurement of patient outcome in arthritis," Arthritis \& Rheumatology, vol. 23, no. 2, pp. 137-145, 1980.

[4] P. M. J. Welsing, A. M. van Gestel, H. L. Swinkels, L. A. L. M. Kiemeney, and P. L. C. M. van Riel, "The relationship between disease activity, joint destruction, and functional capacity over the course of rheumatoid arthritis," Arthritis \& Rheumatology, vol. 44, no. 9, pp. 2009-2017, 2001.

[5] K. M. Pauley, M. Satoh, A. L. Chan, M. R. Bubb, W. H. Reeves, and E. K. L. Chan, "Upregulated miR-146a expression in peripheral blood mononuclear cells from rheumatoid arthritis patients," Arthritis Research \& Therapy, vol. 10, no. 4, article no. R101, 2008.

[6] T. Kobayashi, G. Papaioannou, F. Mirzamohammadi et al., "Early postnatal ablation of the microRNA-processing enzyme, Drosha, causes chondrocyte death and impairs the structural integrity of the articular cartilage," Osteoarthritis and Cartilage, vol. 23, no. 7, pp. 1214-1220, 2015.

[7] S. Thanapati, M. Ganu, P. Giri et al., "Impaired NK cell functionality and increased TNF- $\alpha$ production as biomarkers of chronic chikungunya arthritis and rheumatoid arthritis," Human Immunology, vol. 78, no. 4, pp. 370-374, 2017.

[8] H. Zhang and W. Xiao, "TNFR1 and TNFR2 differentially mediate TNF- $\alpha$-induced inflammatory responses in rheumatoid arthritis fibroblast-like synoviocytes," Cell Biology International, vol. 41, no. 4, pp. 415-422, 2017.

[9] B. Chen, Y. Liu, Y. Zhang, J. Li, K. Cheng, and L. Cheng, "IL-21 Is Positively Associated with Intervertebral Disc Degeneration by Interaction with TNF- $\alpha$ Through the JAK-STAT Signaling Pathway," Inflammation, vol. 40, no. 2, pp. 612-622, 2017.

[10] D. Y. Chen, Y. M. Chen, H. H. Chen, C. W. Hsieh, C. C. Lin, and J. L. Lan, "Increasing levels of circulating Th17 cells and interleukin-17 in rheumatoid arthritis patients with an inadequate response to anti-TNF-alpha therapy," Arthritis Research \& Therapy, vol. 13, no. 4, p. R126, 2011.

[11] J. Sharma, S. Bhar, and C. S. Devi, "A review on interleukins: The key manipulators in rheumatoid arthritis," Modern Rheumatology, vol. 27, no. 5, pp. 723-746, 2017.
[12] Y. H. Huh, G. Lee, W.-H. Song, J.-T. Koh, and J.-H. Ryu, "Crosstalk between FLS and chondrocytes is regulated by HIF$2 \alpha$-mediated cytokines in arthritis," Experimental \& molecular medicine, vol. 47, p. e197, 2015.

[13] A. C. Araujo-Pires, C. F. Francisconi, C. C. Biguetti et al., "Simultaneous analysis of thelper subsets (Th1, Th2, Th9, Th17, Th22, Tfh, Tr1 and Tregs) markers expression in periapical lesions reveals multiple cytokine clusters accountable for lesions activity and inactivity status," Journal of Applied Oral Science, vol. 22, no. 4, pp. 336-346, 2014.

[14] M. Hashizume, N. Hayakawa, M. Suzuki, and M. Mihara, "IL6/sIL-6R trans-signalling, but not TNF- $\alpha$ induced angiogenesis in a HUVEC and synovial cell co-culture system," Rheumatology International, vol. 29, no. 12, pp. 1449-1454, 2009.

[15] M. Narazaki, T. Tanaka, and T. Kishimoto, "The role and therapeutic targeting of IL-6 in rheumatoid arthritis," Expert Review of Clinical Immunology, vol. 13, no. 6, pp. 535-551, 2017.

[16] Y.-J. Guo, J. Chen, X.-G. Xiong, D. Wu, H. Zhu, and Q.-H. Liang, "Effect of Bizhongxiao decoction and its dismantled formulae on IL-1 and TNF levels in collagen-induced arthritis in rat synovial joints.", Theoretical biology \& medical modelling, vol. 9, p. 47, 2012.

[17] L. Zhang, Q.-M. Zhang, Y.-G. Wang, D.-L. Yu, and W. Zhang, "The TCM pattern of the six-zang and six-fu organs can be simplified into the pattern of five-zang and one-fu organs," Journal of Traditional Chinese Medicine, vol. 31, no. 2, pp. 147151, 2011.

[18] D. Wu, J. Chen, H. Zhu et al., "UPLC-PDA determination of paeoniflorin in rat plasma following the oral administration of Radix Paeoniae Alba and its effects on rats with collageninduced arthritis," Experimental and Therapeutic Medicine, vol. 7, no. 1, pp. 209-217, 2014.

[19] B. E. Shan, J. Y. Zhang, and Q. X. Li, "Human T cell and monocyte modulating activity of Rhizoma typhonii in vitro," Chinese Journal of Integrative Medicine, vol. 21, no. 10, pp. 768772, 2001.

[20] S.-J. Kim, W.-S. Chung, S.-S. Kim, S.-G. Ko, and J.-Y. Um, “Antiinflammatory effect of Oldenlandia diffusa and its constituent, hentriacontane, through suppression of caspase-1 activation in mouse peritoneal macrophages," Phytotherapy Research, vol. 25, no. 10, pp. 1537-1546, 2011.

[21] Y. Kwon, S. Chung, T. Kim, and M. Park, "Peroxisome Proliferator-activated Receptor- $\gamma$ Agonist Inhibits Proinflammatory Gene Expressions and Cellular Proliferation of Fibroblast Like Synoviocytes from Patients with Rheumatoid Arthritis by Down-regulation of NF-kappaB," The Journal of the Korean Rheumatism Association, vol. 17, no. 2, p. 153, 2010.

[22] Y. Dowlati, N. Herrmann, W. Swardfager et al., "A meta-analysis of cytokines in major depression," Biological Psychiatry, vol. 67, no. 5, pp. 446-457, 2010.

[23] K. Tanabe, R. Matsushima-Nishiwaki, S. Yamaguchi, H. Iida, S. Dohi, and O. Kozawa, "Mechanisms of tumor necrosis factor$\alpha$-induced interleukin-6 synthesis in glioma cells," Journal of Neuroinflammation, vol. 7, article no. 16, 2010.

[24] D. R. Park, R. Ko, S. H. Kwon et al., "FlexPro MD, a Mixture of Krill Oil, Astaxanthin, and Hyaluronic Acid, Suppresses Lipopolysaccharide-Induced Inflammatory Cytokine Production Through Inhibition of NF- $\kappa$ B," Journal of Medicinal Food, vol. 19, no. 12, pp. 1196-1203, 2016.

[25] H. Zhu, Q.-H. Liang, X.-G. Xiong et al., "Anti-inflammatory effects of the bioactive compound ferulic acid contained in oldenlandia diffusa on collagen-induced arthritis in rats," 
Evidence-Based Complementary and Alternative Medicine, vol. 2014, Article ID 573801, 10 pages, 2014.

[26] J. Shi, S.-Y. Jiang, X.-J. Ma, H. Sun, and Y. Zhou, "Studies on seeds germination and seedlings growth of Notopterygium incisum," China Journal of Chinese Materia Medica, vol. 32, no. 18, pp. 1841-1844, 2007.

[27] T. R. Mikuls and J. O'Dell, “The changing face of rheumatoid arthritis therapy: results of serial surveys," Arthritis \& Rheumatology, vol. 43, no. 2, pp. 464-465, 2000.

[28] N. B. Nataraj, J. Krishnamurthy, and B. P. Salimath, “Treatment with anti-NAP monoclonal antibody reduces disease severity in murine model of novel angiogenic protein-induced or ovalbumin-induced arthritis," Clinical \& Experimental Immunology, vol. 171, no. 2, pp. 155-163, 2013.

[29] Q.-M. Zhang and Z.-Y. Sun, "Study on Chemical Constituents of Oldenlandia diffusa," Zhong yao cai = Zhongyaocai = Journal of Chinese medicinal materials, vol. 37, no. 12, pp. 2216-2218, 2014.

[30] H.-Q. Li, Y. Cao, Y.-B. Bai, B.-H. Xia, L.-M. Lin, and D.-F. Liao, "UPLC Fingerprint of Oldenlandia corymbosa," Journal of Chinese Medicinal Materials, vol. 38, no. 4, pp. 735-738, 2015.

[31] S. J. Pragasam, V. Venkatesan, and M. Rasool, "Immunomodulatory and anti-inflammatory effect of p-coumaric acid, a common dietary polyphenol on experimental inflammation in rats," Inflammation, vol. 36, no. 1, pp. 169-176, 2013.

[32] F. Ursini, C. Leporini, F. Bene et al., "Anti-TNF-alpha agents and endothelial function in rheumatoid arthritis: A systematic review and meta-analysis," Scientific Reports, vol. 7, no. 1, article no. 5346, 2017.

[33] M. A. Alam, N. Subhan, H. Hossain et al., "Hydroxycinnamic acid derivatives: A potential class of natural compounds for the management of lipid metabolism and obesity," Journal of Nutrition and Metabolism, vol. 13, no. 1, article no. 27, 2016.

[34] K. Schinnerling, J. C. Aguillón, D. Catalán, and L. Soto, “The role of interleukin- 6 signalling and its therapeutic blockage in skewing the T cell balance in rheumatoid arthritis," Clinical \& Experimental Immunology, vol. 189, no. 1, pp. 12-20, 2017.

[35] S. Hirohata, A. Abe, A. Murasawa, T. Kanamono, T. Tomita, and H. Yoshikawa, "Differential effects of IL-6 blockade tocilizumab and TNF inhibitors on angiogenesis in synovial tissues from patients with rheumatoid arthritis," Modern Rheumatology, vol. 27, no. 5, pp. 766-772, 2017.

[36] P. Welsh, K. Tuckwell, I. B. McInnes, and N. Sattar, "Effect of IL-6 receptor blockade on high-sensitivity troponin T and NTproBNP in rheumatoid arthritis," Atherosclerosis, vol. 254, pp. 167-171, 2016.

[37] S. Li, Z. Wu, L. Li, and X. Liu, "Interleukin-6 (IL-6) receptor antagonist protects against rheumatoid arthritis," Medical Science Monitor, vol. 22, pp. 2113-2118, 2016.

[38] L. I. Filippin, R. Vercelino, N. P. Marroni, and R. M. Xavier, "Redox signalling and the inflammatory response in rheumatoid arthritis," Clinical \& Experimental Immunology, vol. 152, no. 3, pp. 415-422, 2008.

[39] J. Hillen, C. Geyer, M. Heitzmann et al., "Structural cartilage damage attracts circulating rheumatoid arthritis synovial fibroblasts into affected joints," Arthritis Research \& Therapy, vol. 19, no. 1, article no. 40, 2017.

[40] E. Ku, V. Pedoia, M. Tanaka et al., "Evaluating radiocarpal cartilage matrix changes 3-months after anti-TNF treatment for rheumatoid arthritis using MR T1 $\rho$ imaging," Journal of Magnetic Resonance Imaging, vol. 45, no. 5, pp. 1514-1522, 2017.
[41] S.-T. Wei, Y.-H. Sun, S.-H. Zong, and Y.-B. Xiang, "Serum levels of IL- 6 and TNF- $\alpha$ may correlate with activity and severity of rheumatoid arthritis," Medical Science Monitor, vol. 21, pp. 4030-4038, 2015.

[42] H.-J. Shin, M.-S. Choi, N.-H. Ryoo et al., "Manganese-mediated up-regulation of HIF- $1 \alpha$ protein in Hep2 human laryngeal epithelial cells via activation of the family of MAPKs," Toxicology in Vitro, vol. 24, no. 4, pp. 1208-1214, 2010.

[43] B. B. Scott, P. F. Zaratin, A. G. Gilmartin et al., "TNF- $\alpha$ modulates angiopoietin-1 expression in rheumatoid synovial fibroblasts via the NF- $\kappa \mathrm{B}$ signalling pathway," Biochemical and Biophysical Research Communications, vol. 328, no. 2, pp. 409414, 2005.

[44] H. Xu, Y. He, X. Yang et al., "Anti-malarial agent artesunate inhibits TNF- $\alpha$-induced production of proinflammatory cytokines via inhibition of NF- $\kappa \mathrm{B}$ and PI3 kinase/Akt signal pathway in human rheumatoid arthritis fibroblast-like synoviocytes," Rheumatology, vol. 46, no. 6, pp. 920-926, 2007.

[45] S. N. Lauder, S. M. Carty, C. E. Carpenter et al., "Interleukin$1 \beta$ induced activation of nuclear factor- $\kappa$ b can be inhibited by novel pharmacological agents in osteoarthritis," Rheumatology, vol. 46, no. 5, pp. 752-758, 2007. 


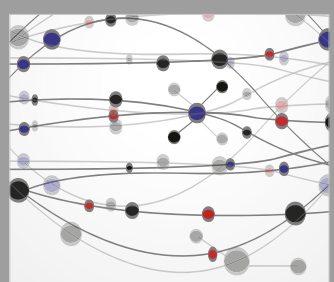

The Scientific World Journal
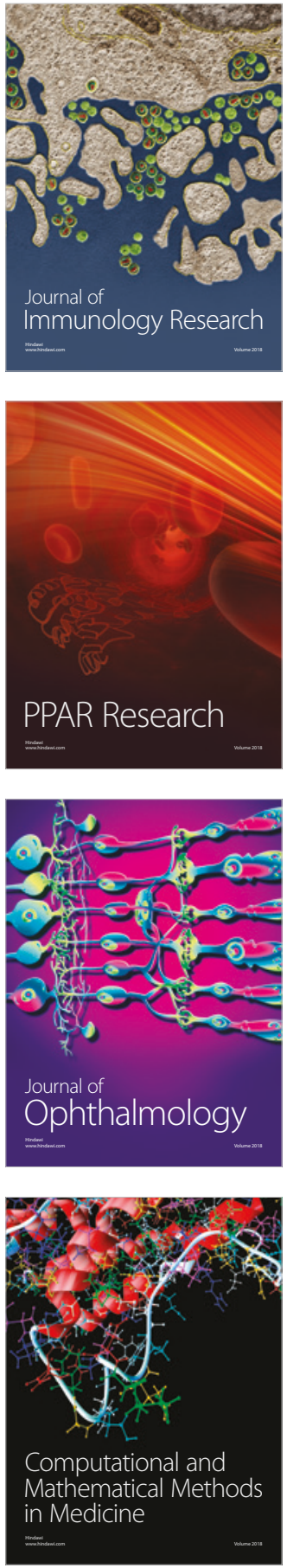

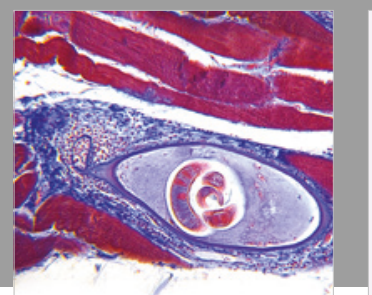

Gastroenterology Research and Practice

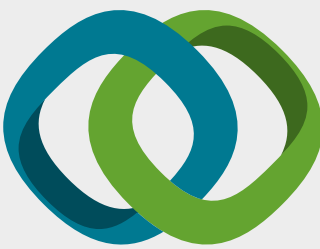

\section{Hindawi}

Submit your manuscripts at

www.hindawi.com
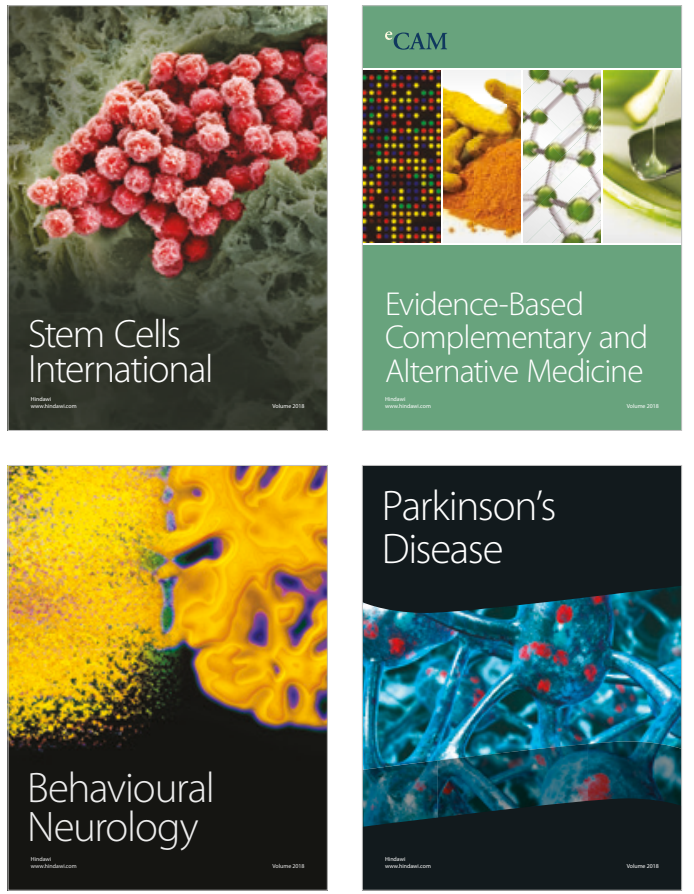

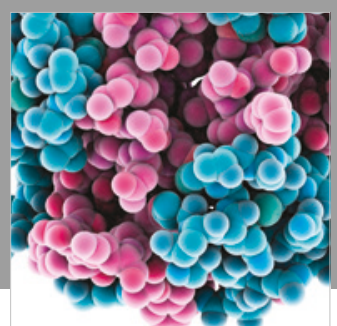

ournal of

Diabetes Research

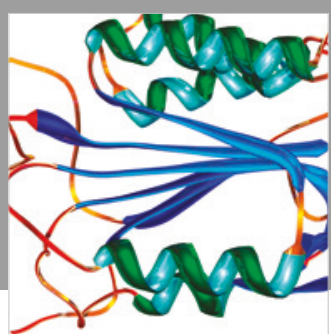

Disease Markers
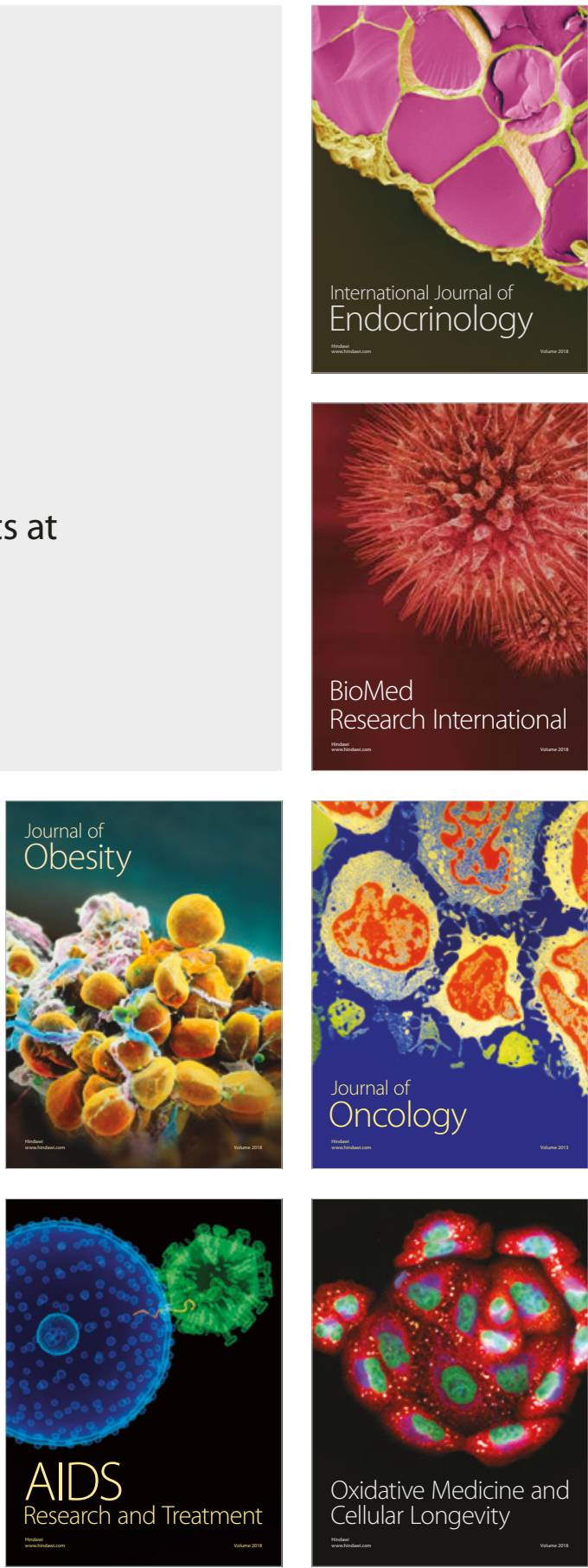